\title{
Un marché de l'emploi intégré ? L'emploi frontalier et ses dimensions socioéconomiques
}

\author{
Rachid Belkacem/Isabelle Pigeron-Piroth
}

Un des éléments les plus caractéristiques de la Grande Région SaarLorLux est l'importance des flux de travailleurs frontaliers qui ont lieu entre ses diverses composantes. Questionner les réalités de vie quotidienne et les constructions politiques au sein de cet espace passe nécessairement par une analyse de ces flux de travail frontalier, véritable trait d'union entre les composantes de la Grande Région SaarLorLux.

Les dernières décennies ont vu une croissance sans précédent du nombre de travailleurs frontaliers dans la Grande Région SaarLorLux, particulièrement à destination du Luxembourg. Ils sont 213400 individus (en 2012) à quitter tous les jours (ou au moins une fois par semaine) leur pays de résidence pour se rendre sur leur lieu de travail situé dans un des pays voisins. De nombreux travaux ont été réalisés pour expliquer ce développement, analyser ses dimensions socioéconomiques, cerner ses effets sur le développement économique et social des territoires (cf. Belkacem/Pigeron-Piroth 2012a). Il ressort de ces recherches que le développement des mobilités n'est pas sans conséquence sur les dynamiques des marchés du travail régionaux, sur les systèmes de formation, mais également sur les dynamiques de structuration de l'espace. La question développée dans cette contribution sera la suivante: pour autant, le travail frontalier contribue-t-il à l'intégration des différentes composantes régionales de la Grande Région SaarLorLux à un même grand espace transfrontalier ? Les éléments permettant d'y apporter une réponse s'articulent autour de deux parties. Dans une première partie, nous commençons par souligner la réalité $d u$ travail frontalier et sa structuration au sein de la Grande Région SaarLorLux. Ensuite, nous montrons, dans un deuxième temps, que l'intégration transfrontalière en matière d'emploi, bien qu'en cours de réalisation, reste limitée à plusieurs égards. 


\section{L'importance et la structuration du travail frontalier dans la Grande Région SaarLorLux}

Notre cadre d'étude, la Grande Région SaarLorLux, possède la spécificité de se trouver aux marges de différents territoires nationaux. Léloignement des centres de décisions nationaux respectifs, mais la proximité géographique (ainsi que culturelle, linguistique, etc.) avec les territoires des pays voisins confèrent à cet espace transfrontalier une certaine dynamique et une particularité incontestable: l'importance des flux de travail frontalier. Forte de ses nombreux travailleurs frontaliers, la Grande Région SaarLorLux est le terrain de flux, relations et interdépendances en tout genre (cf. p.ex. Evrard/Schulz, Wille et Boesen/ Schnuer dans cet ouvrage) qui lui donnent une légitimité et un sens qu'il convient de détailler à présent.

\subsection{Des origines historiques et des éléments explicatifs}

Le travail frontalier a des origines anciennes au sein de cet espace, en lien avec les richesses de son sous-sol (fer, charbon). Le bassin sidérurgique et minier ne s'arrête en effet pas aux frontières nationales. Les savoir-faire et les compétences industrielles s'employaient déjà de l'autre côté des frontières durant la période d'industrialisation (cf. Helfer dans cet ouvrage). Le travail frontalier a permis de faire face à des besoins de main-d'œuvre en période de surcroit d'activité, comme dans la période de reconstruction après la Première Guerre Mondiale. $\mathrm{Au}$ Luxembourg par exemple, le régime de carte frontière en vigueur dans les années 1920 garantissait une certaine souplesse, à la fois aux autorités luxembourgeoises et aux travailleurs concernés. A condition de résider en France, Belgique ou Allemagne, à proximité directe des frontières luxembourgeoises (selon une liste de communes établie par l'arrêté ministériel du 9 février 1921), les frontaliers pouvaient venir travailler au Luxembourg avec une simple carte comportant leur identité, adresse et profession (cf. Sauer/Scuto 2012). Le développement du travail frontalier peut également s'expliquer par des éléments de proximité géographique et culturelle, mais également linguistique (de nombreux Lorrains habitant à proximité des frontières allemandes sont bilingues, et la plupart des Luxembourgeois parlent allemand et français en plus de leur langue nationale). Mais d'un autre côté, les différences entre les pays sont aussi un élément explicatif du travail frontalier. Les divergences de développement ou d'organisation de l'activité économique entre les territoires sont un facteur de croissance du travail frontalier : certains ayant des besoins de main-d'œuvre (comme le Luxembourg), d'autres des disponibilités en termes de main-d'œuvre (comme la Lorraine). L'existence de différentiels de part et d'autre des frontières (dans les coûts du travail, les niveaux de rémunération ou encore les coûts de 
l'immobilier) alimentent également toute la dynamique liée au développement du travail frontalier. Des rémunérations plus élevées au Luxembourg et en Allemagne rendent le travail dans ces pays plus attractif. Alors que les coûts de l'immobilier très élevés ont plutôt un effet «répulsif».

Par ailleurs, le statut de travailleur frontalier fait l'objet d'une construction sociale. Des règles ou encore des conventions (conventions fiscales bilatérales pour le paiement des impôts, définition du statut de travailleur frontalier du point de vue de la sécurité sociale, etc.) permettent de l'encadrer et de le définir: «Le terme de travailleur frontalier désigne toute personne qui exerce une activité salariée ou non salariée dans un Etat membre et qui réside dans un autre Etat membre où elle retourne en principe chaque jour ou au moins une fois par semaine» (règlement [CE] 883/2004). Ce contexte est d'ailleurs évolutif et des mises à jour sont régulièrement effectuées dans la coordination afin de tenir compte des changements dans les législations nationales. Ainsi, ce règlement (CE) 883/2004 remplace le précédent (règlement [CE] 1408/71) qui définissait depuis 1971 le statut de travailleur frontalier.

\subsection{Un phénomène ancré dans les économies locales}

D'après l'Observatoire Interrégional de l'Emploi (OIE), les travailleurs frontaliers de la Grande Région SaarLorLux constituent le quart des flux de frontaliers de l'Union Européenne (UE27; cf. OIE 2012 : 118). La carte 1 révèle l'attractivité du Luxembourg, principale destination des travailleurs frontaliers de la Grande Région SaarLorLux. La Lorraine quant à elle, est le principal «fournisseur» de travailleurs frontaliers au sein de la Grande Région SaarLorLux. Aujourd'hui, plus de 100000 Lorrains ont leur emploi de l'autre côté des frontières, dont les trois-quarts au Luxembourg (près de $20 \%$ en Allemagne et $5 \%$ en Belgique). Cela signifie que près d'un Lorrain sur dix exerce son activité dans un pays voisin. Le Luxembourg, pour sa part, a recours à la fois au travail frontalier (près de $44 \%$ des salariés en 2013 ) et à l'immigration ( $27,5 \%$ des salariés), afin de satisfaire ses besoins de main-d'œuvre (cf. graphique 1).

Notons également que sur ces vingt dernières années, la main-d'œuvre étrangère au Luxembourg a beaucoup augmenté. Les salariés frontaliers ont en effet été multipliés par près de quatre et les immigrés salariés par près de deux. Au-delà de ces quelques chiffres et évolutions, il importe de souligner la situation d'interdépendance entre les territoires de la Grande Région SaarLorLux, et le poids que le travail frontalier a pris dans les économies locales. Si à l'échelle de la Lorraine, un dixième des actifs va travailler outre-frontière, dans certaines zones d'emploi comme celle de Longwy, ancien bastion sidérurgique du nord de la région, contigu avec le Luxembourg et la Belgique, c'est près de la moitié des 


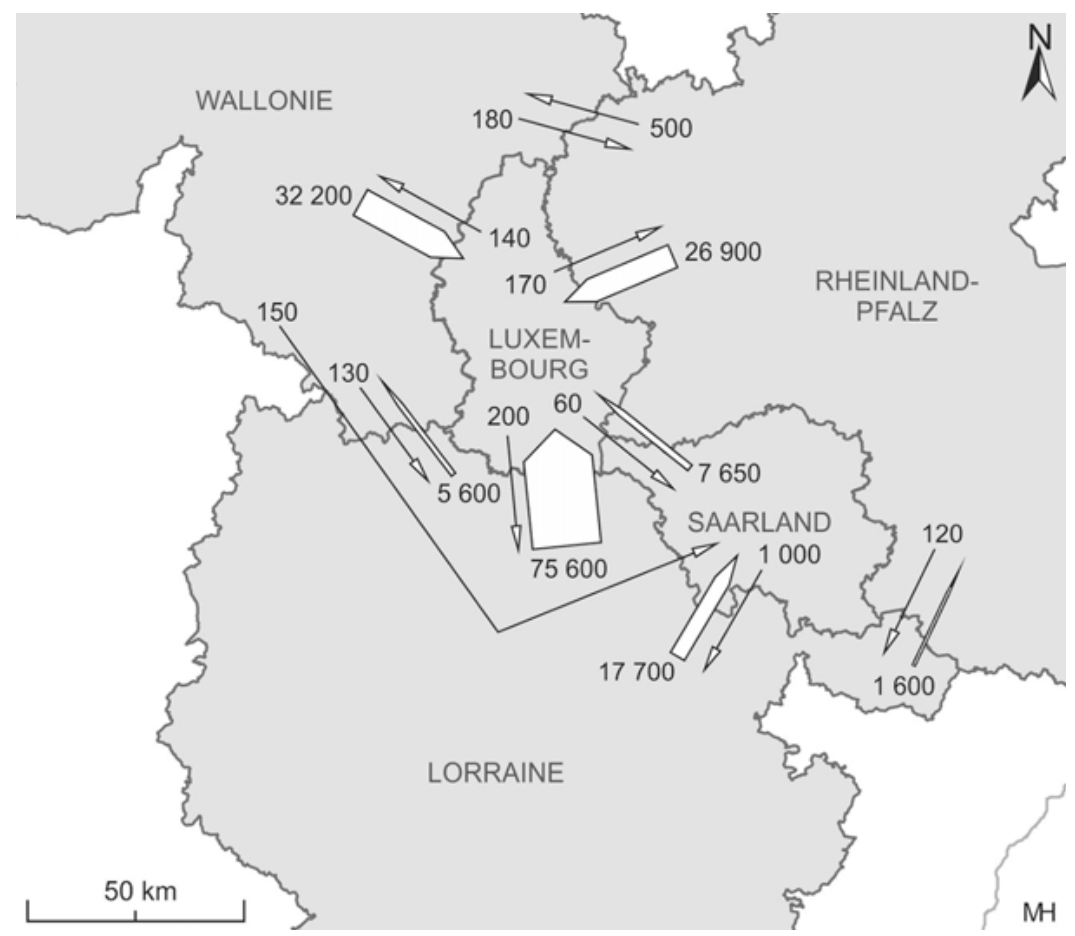

Carte 1: Les flux de travail frontalier au sein de la Grande Région SaarLorLux en 2011, d'après les données INSEE, IGSS, IWEPS, Statistisches Amt Saarland, Statistisches Landesamt Rheinland-Pfalz (Cartographie: Malte Helfer)

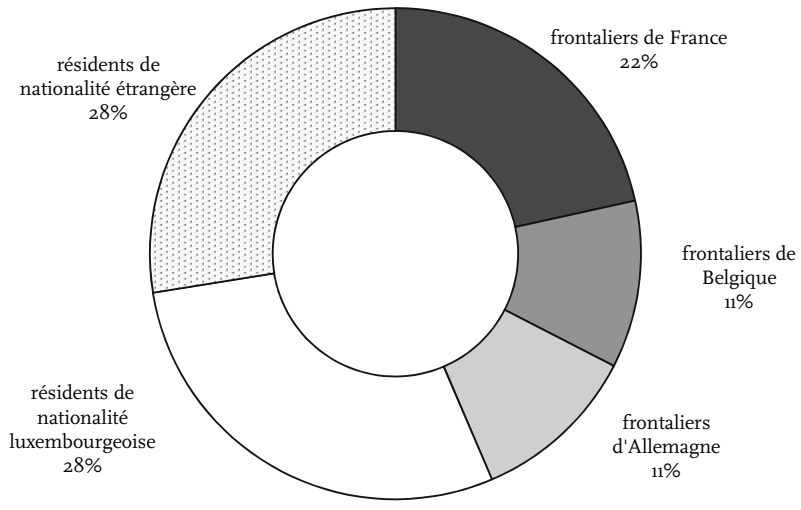

Graphique 1: Structure de l'emploi salarié au Luxembourg (31 mars 2013)

Université du Luxembourg, d'après les données IGSS/CCSS 
actifs locaux qui travaille dans le pays voisin (49\% en 2011, INSEE). Quant aux petites communes directement frontalières, certaines voient entre 70 et $80 \%$ de leur population active occupée dans le pays voisin. C'est par exemple le cas des communes françaises d'Evrange, Rédange, Hagen et Zoufftgen d'après les données INSEE.

Par ailleurs, le phénomène se structure. L'INSEE parle d'un ancrage du travail frontalier dans l'économie lorraine, à la fois par une adaptabilité des frontaliers aux évolutions économiques et aux besoins des marchés, et par leur vieillissement.

Ainsi, les spécialisations sectorielles des frontaliers ne se figent pas mais s'adaptent aux évolutions économiques luxembourgeoises. Et signe de maturité, le phénomène frontalier étend son emprise à l'ensemble des classes d'âge, la primauté des jeunes actifs s'estompant quelque peu. (Moreau/Neiss 2007 : 1)

Les frontaliers vieillissent et connaissent des carrières outre-frontière, signe que le travail frontalier est envisagé de manière durable. La comparaison des principales caractéristiques des travailleurs frontaliers occupés au Luxembourg en 1995 et en 2013 va dans ce sens, puisque le vieillissement de la main-d'œuvre frontalière au Luxembourg a été plus important (+ 5,6 ans) que celui de la maind'œuvre immigrée $(+4,8$ ans) et surtout nationale $(+3,1$ ans) (d'après les données de la sécurité sociale luxembourgeoise). En fait, ce sont surtout les femmes frontalières qui ont connu une forte hausse de leur âge moyen (près de sept ans de plus). Malgré une certaine féminisation ces vingt dernières années, les travailleurs frontaliers restent les salariés les moins féminisés de tous $34,5 \%$ de femmes parmi les frontaliers contre $40 \%$ pour l'ensemble des salariés. On notera même que seuls 30,5\% des frontaliers venant de Belgique sont des femmes). L'évolution 1995-2013 nous montre aussi les modifications dans les profils, révélant dans quelle mesure ils se sont adaptés aux besoins de l'emploi luxembourgeois. En 1995, près d'un frontalier sur quatre $(24 \%)$ travaillait dans les industries manufacturières, $16 \%$ dans le commerce et $15 \%$ dans les services aux entreprises (selon NACE Rev1). Vingt ans plus tard, le trio de tête est inversé : le principal secteur d'activité des frontaliers est celui des services aux entreprises (22\%), suivi du commerce (15\%) et des industries manufacturières (13\%).

Cette évolution socioprofessionnelle et sectorielle du travail frontalier s'accompagne d'autres inflexions, notamment dans les caractéristiques des déplacements. La longueur des trajets est, certes, très variable, certains frontaliers parcourant à peine quelques kilomètres, alors que d'autres sont beaucoup plus éloignés. La moyenne pour les frontaliers occupés au Luxembourg s'établit à 44 kilomètres en 2010 , une distance parcourue en 53 minutes pour rejoindre leur lieu de travail (trajet aller), avec des différences en fonction du pays de résidence (cf. Schmitz/Drevon/Gerber 2012). Fait intéressant, on assiste à 
la fois à un renforcement des principaux pôles de résidence à proximité des frontières (comme Thionville, Villerupt, Longwy en France, ou Arlon en Belgique), mais aussi à l'apparition de nouveaux pôles secondaires plus éloignés des frontières luxembourgeoises (Trèves ou encore Metz), bien desservis par les infrastructures routières ou ferroviaires. L'aire d'attraction du Luxembourg tend à s'étendre bien au-delà des territoires directement limitrophes. Le bassin d'emploi du Luxembourg déborde en effet largement ses frontières et les limites de la Grande Région SaarLorLux, afin de trouver de la main-d'œuvre qualifiée et des profils de plus en plus pointus. Il n'est pas rare de trouver des frontaliers «éloignés » parcourant plus de $100 \mathrm{~km}$ pour rejoindre leur lieu de travail au Luxembourg. Ils sont par exemple près de 400 à venir de Nancy et de son agglomération en 2013. Il se peut que ces travailleurs frontaliers ne pendulent pas quotidiennement et disposent donc de plusieurs résidences. En effet, la définition de la sécurité sociale considère que le travailleur frontalier effectue un aller-retour au moins une fois par semaine, et donc pas nécessairement tous les jours. Ce sujet de la multirésidence est révélateur de nouvelles formes de travail frontalier (navettes non-quotidiennes vers la résidence principale), et de nouveaux rapports à l'espace, mais également à la famille (cf. Pigeron-Piroth 2014). C'est tout à la fois l'éloignement entre lieu de résidence et lieu de travail, la présence d'un ménage déjà constitué (un conjoint actif, des enfants scolarisés, la propriété d'un bien immobilier, etc.), qui va expliquer cette résidence multiple. Kaufmann (2008: 21) souligne justement ce «paradoxe de la mobilité » en montrant que «Les nouveaux moyens de déplacement ne sont donc pas le signe d'une «hypermobilité » croissante. C'est au contraire pour préserver notre environnement familier et nos ancrages que nous choisissons maintes manières de nous déplacer vite et loin. » Ces travailleurs se déplacent beaucoup « dans le but de rester socialement enracinés ». Dans le cas de la Grande Région SaarLorLux, ces mobilités de longue distance sont à la fois révélatrices de l'agrandissement du «périmètre d'attraction du Luxembourg », et de l'importance des ancrages. La présence de frontières et des différentiels qui y sont liés (en matière de prix immobiliers, mais aussi de système scolaire, de pratique linguistique, etc.) tend à favoriser ces mobilités de longue distance plutôt qu'un rapprochement du lieu de travail.

\subsection{Une grande variété de profils}

Les profils des travailleurs frontaliers occupés au Luxembourg sont hétérogènes et les secteurs dans lesquels ils ont un emploi diffèrent dans l'espace. Ces multiples profils révèlent une structuration spatiale du marché du travail frontalier. Les frontaliers d'Allemagne et de Belgique sont plus souvent concernés par un emploi dans les activités financières (respectivement 15 et 15,5\%) par rapport 
à ceux venant de France $(12 \%$ dans ce cas). Alors que ces derniers sont plus nombreux dans l'industrie manufacturière (14\%) mais aussi dans le commerce (16\%) ou l'intérim, révélant des niveaux de diplôme en moyenne inférieurs par rapport aux autres frontaliers. Au sein d'un même pays, on notera également des différences de profil en fonction des lieux de résidence. En France, les frontaliers venant de Metz sont nombreux à travailler dans la finance (plus de $20 \%$ ). Alors que Longwy est encore marqué par son passé industriel, ce qui se retrouve dans les secteurs d'activité de sa population: moins de $10 \%$ des frontaliers originaires du canton de Longwy travaillent dans la finance au Luxembourg, alors que $16 \%$ sont occupés dans l'industrie.

Les besoins des différents marchés ne sont par ailleurs pas identiques. Les travailleurs frontaliers Lorrains ayant un emploi en Allemagne et ceux ayant un emploi au Luxembourg ne travaillent pas dans les mêmes secteurs (cf. graphique 2). Dans l'ensemble, l'emploi des Lorrains en Sarre est très fortement masculin, peu diplômé et à $45 \%$ industriel (industrie automobile principalement). Alors que les Lorrains occupés au Luxembourg sont plus diplômés dans l'ensemble, comptent davantage de femmes (même si elles restent minoritaires) et sont nettement moins concernés par un emploi dans l'industrie (moins de $15 \%$; secteur C). Commerce $(\mathrm{G})$, finance $(\mathrm{K})$ et interim/nettoyage $(\mathrm{N})$ sont leurs principaux secteurs d'activité.

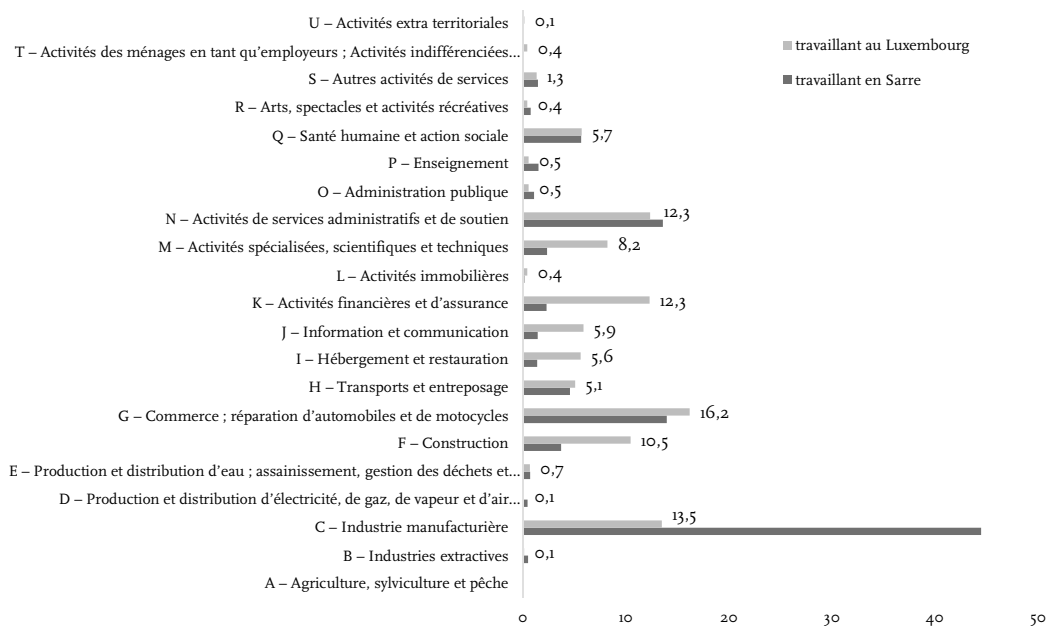

Graphique 2: Les secteurs d'activité (NACE-REV2) des travailleurs frontaliers Lorrains occupés en Sarre et au Luxembourg (en \%) (2011/12), Université du Luxembourg, d'après les données IGSS/CCSS (pour les frontaliers occupés au Luxembourg, données du 31 mars 2012), données OIE (pour les frontaliers occupés en Sarre, données du zo juin 2011) 
Cette analyse permet de livrer deux principales caractéristiques du travail frontalier. La première concerne sa relative concentration géographique, avec cependant un mouvement d'extension des zones de résidence des travailleurs frontalier. L'intensification de ce mouvement va dépendre de plusieurs paramètres, comme les conditions de mobilité en lien avec les infrastructures de déplacement, mais aussi les besoins en qualifications de plus en plus pointus, sans oublier des considérations d'ordre social et familial. La seconde caractéristique se rapporte à la relative spécialisation géographique du travail frontalier. On relève en effet des disparités géographiques dans les modes d'usage de ces travailleurs frontaliers en fonction de leur lieu de résidence et de travail. De ces observations empiriques, il en découle que le travail frontalier ne permet pas seulement d'effectuer un ajustement quantitatif (en effectifs) entre besoins et disponibilité de main-d'œuvre, de part et d'autre de la frontière. Il permet aussi, et sans doute de plus en plus, un ajustement qualitatif entre la nature des besoins et les qualifications des travailleurs (cf. Belkacem/Pigeron-Piroth 2012b).

La structuration du travail frontalier dont nous faisons état dans cette première partie est ainsi complexe. Elle ne concerne pas seulement sa croissance et sa durabilité. Elle touche également à ses fonctions, appréhendées par la diversification de ses formes. A côté de contrats de travail plutôt classiques entre un employeur et un salarié sont apparues des formes particulières d'emploi, et notamment le travail intérimaire transfrontalier, c'est-à-dire une activité temporaire exercée de l'autre côté de la frontière (cf. Belkacem/Pigeron-Piroth 2014).

\subsection{Les effets et enjeux du travail frontalier sur le développement économique et territorial}

Par son importance, son caractère structurel et son fort ancrage territorial, le travail frontalier n'est pas sans effets sur le développement économique et social des territoires impliqués des deux côtés de la frontière (territoire de résidence et territoire d'emploi de ces travailleurs). Nos analyses porteront ici principalement sur le nord de la Lorraine et le Luxembourg, territoires étant les plus imbriqués en matière d'emploi, car les plus dépendants vis-à-vis du travail frontalier.

Pour les territoires de résidence, le travail frontalier alimente trois principales dynamiques de développement. Tout d'abord, il est un amortisseur des crises industrielles successives. Il a permis d'atténuer les effets négatifs des restructurations particulièrement dans les territoires du nord de la Lorraine comme par exemple dans le bassin d'emploi de Longwy. Pour rappel, 26 hauts-fourneaux étaient en activité sur ce territoire. Plus aucun aujourd'hui. A la grande époque de l'âge d'or de l'industrie lorraine et jusqu'aux années 1970, cette activité occupait 30 o०o personnes sur tout le nord de la Meurthe-et-Moselle (110 o०० pour l'ensemble de la Lorraine). La zone d'emploi de Longwy est celle qui a subi les 
plus fortes mutations industrielles et qui caractérise aujourd'hui une des plus fortes dépendances au travail frontalier. A la suite de la fermeture de ces usines, dans ce contexte de mono-industrie, le travail frontalier offre une alternative d'emploi à de nombreux actifs venant de perdre leur emploi ou n'ayant pas de perspectives d'insertion professionnelle sur ce territoire. Ce faisant, le travail frontalier a contribué à maintenir sur place de nombreux ménages, leur offrant ainsi des perspectives de débouchés juste de l'autre côté de la frontière. Sur ce territoire, aujourd'hui, près d'un actif sur deux a son activité professionnelle de l'autre côté de la frontière. En 2011, près de 22000 actifs résidant dans la zone d'emploi de Longwy travaillent de l'autre côté des frontières, principalement au Luxembourg (cf. INSEE). Par ailleurs, la population locale progresse à nouveau, alors que ce n'était plus le cas depuis la fin des années soixante. Et l'attractivité grandissante de ces espaces géographiques limitrophes se manifeste par une hausse $d u$ prix du foncier ces dernières décennies.

Le travail frontalier a également contribué à l'élévation des niveaux moyens de revenus dans les territoires de résidence des frontaliers. Certains cantons proches du Luxembourg (Cattenom, Thionville) font ainsi partie des $5 \%$ possédant le revenu moyen le plus élevé de toute la France (cf. Thanry 2012). A ces revenus s'ajoutent les prestations familiales, plus élevées au Luxembourg qu'en France et qui contribuent à augmenter le pouvoir d'achat des ménages concernés, pouvoir d'achat qui sera pour partie réinjecté localement.

A ce titre, le travail frontalier impulse une nouvelle dynamique économique, avec l'émergence d'activités liées aux services ou aux loisirs, et plus généralement à l'économie résidentielle (cf. Belkacem/Pigeron-Piroth 2012c). Ce nouveau type de dynamique économique tranche avec le passé industriel de ces territoires. Il vise la satisfaction des besoins de personnes présentes ou passagères dans la zone, plus nombreuses du fait de la dynamique liée aux frontières (mobilités de travail, de résidence, d'achats, de loisirs, etc.). A titre d'exemple, l'un des plus grands centres commerciaux de France implanté à Mont-SaintMartin, la création d'un complexe cinématographique, d'une médiathèque hightech, ou encore la construction d'un golf sur les anciennes friches industrielles, caractérisent cette nouvelle orientation dans le développement économique $\mathrm{du}$ territoire. En y ajoutant les emplois liés aux fonctions de santé et action sociale, d'administration publique, de distribution, d'éducation et de services de proximité, ces activités liées à l'économie résidentielle représentent aujourd'hui selon l'INSEE environ $56 \%$ de l'emploi local pour les territoires à proximité directe du Luxembourg (zones d'emploi de Meuse du Nord, Longwy, Briey et Thionville; cf. François/Moreau 2010). D'après certains auteurs, cette base résidentielle représente en moyenne $40 \%$ des revenus qu'un territoire peut tirer de l'extérieur et qui impulsent son développement (cf. Davezies/Talandier 2010). Alors que seul un quart des revenus basiques des zones d'emploi françaises est de nature productive. 
Par ailleurs, le travail frontalier est un facteur de développement économique pour les territoires d'emploi. La forte croissance économique du Luxembourg n'aurait pu être possible sans le recours aux travailleurs frontaliers. Certains secteurs de l'économie luxembourgeoise n'ont pu se développer que par cet apport de main-d'œuvre, la population active nationale ne suffisant pas, et travaillant pour beaucoup dans le secteur public et semi-public ( $42 \%$ des salariés de nationalité luxembourgeoise y travaillent en mars 2008 où ils représentent $87 \%$ de la main-d'œuvre de ces secteurs) (cf. Pigeron-Piroth 2009). Le secteur des industries manufacturières (NACE Rev1) est en 2013 le secteur comptant la plus forte proportion de frontaliers parmi ses salariés (62\%). Par ailleurs, les services aux entreprises, la construction et le commerce comptent plus de la moitié de frontaliers parmi leurs salariés. Mais le travail frontalier contribue également à combler les besoins en termes de qualifications et de compétences. Une étude menée à l'échelle de la Grande Région SaarLorLux montre que les travailleurs frontaliers sont plus diplômés que les actifs qui résident et travaillent sur place (cf. Löh 2011). Les frontaliers venant de Wallonie sont d'ailleurs les plus diplômés : près de la moitié ont un diplôme universitaire. Ceci représente un avantage important pour le Luxembourg qui dispose d'une main-d'œuvre formée et diplômée sans en supporter les coûts.

Selon les conventions bilatérales signées entre le Luxembourg et les pays voisins, les travailleurs frontaliers occupés au Grand-Duché sont imposés à la source dans ce pays. Ces prélèvements correspondent à une importante manne financière qui permet de soutenir tant la dynamique économique que la redistribution sociale. De plus, les travailleurs frontaliers sont également des consommateurs dans leur pays de travail, souvent motivés par le gain de temps (raison principale évoquée par $51 \%$ des frontaliers interrogés) ou l'offre de produits (pour $30 \%$ ) (cf. Zanardelli/Genevois/Schuller 2012). Ceci remet en cause les idées selon lesquelles les frontaliers ne consommeraient dans leur pays d'emploi que des produits moins chers comme l'essence, le tabac ou encore l'alcool (cf. aussi Wille dans cet ouvrage). En 2007, la dépense sur le territoire luxembourgeois des 135000 travailleurs frontaliers a été estimée à 1 milliard 220 millions d'euros, ce qui correspond à une dépense annuelle moyenne d'environ 9 oo euros par frontalier. Ces travailleurs auraient consacré en effet $37 \%$ de ce montant moyen à des dépenses qualifiées de «substituables courantes », c'est-àdire des dépenses qui auraient pu être effectuées sur leur territoire de résidence (vêtements, courses ou encore les loisirs). Une part de $22 \%$ aurait été consacrée à des dépenses substituables non courantes (voyage, voiture), $11 \%$ à des dépenses contraintes par l'activité professionnelle (restauration, transport, etc.) et le reste, soit $30 \%$, à des dépenses relatives à des produits soumis à accises comme le tabac par exemple.

L'ensemble des enseignements empiriques développés dans cette première partie témoignent de l'interconnexion entre les territoires permise par le travail 
frontalier et de l'émergence d'un marché du travail transfrontalier. Le travail frontalier donne à ce titre une certaine réalité à la Grande Région SaarLorLux. Mais peut-on pour autant parler d'intégration de ces territoires à un même grand espace transfrontalier?

\section{Une intégration transfrontalière limitée}

Le travail frontalier a des apports indéniables pour le développement des territoires frontaliers, comme nous l'avons vu. Mais il peut aussi être source d'effets pervers, notamment du fait des différences institutionnelles entre les territoires.

\subsection{Des effets pervers du travail frontalier}

Le travail frontalier incite par exemple à une «fuite» des plus diplômés vers le Luxembourg, plus attractif du point de vue des salaires. Certaines entreprises sur les territoires de résidence ne disposent alors plus des compétences nécessaires à leur développement ou n'arrivent plus à stabiliser leur personnel. Il existe par ailleurs une différence dans le niveau des charges sociales entre les pays, rendant le Luxembourg également plus attractif pour les entreprises.

Une certaine disparité sociale et des «poches de pauvreté » peuvent apparaitre au sein des territoires frontaliers (cf. Thanry 2012). A côté des travailleurs frontaliers aux revenus moyens élevés, se trouve une population beaucoup moins bien lotie, souffrant davantage de la hausse des prix immobiliers, plus concernée par le revenu de solidarité active (RSA) et le logement social. La zone d'emploi de Longwy concentre à ce titre la plus forte proportion de travailleurs pauvres de toute la Lorraine.

Un autre effet pervers concerne les difficultés des territoires de résidence face aux défis urbains, démographiques ou sociaux posés par le travail frontalier. Comme les frontaliers paient leur impôt au Luxembourg (prélèvement à la source), les collectivités territoriales ne bénéficient pas des retombées fiscales du travail frontalier, alors qu'elles voient leur population s'accroître. Les investissements (écoles, équipements, etc.) répondant aux besoins de cette population croissante peuvent être lourds pour certaines de ces communes (notamment les plus petites).

Par ailleurs, les pays comme le Luxembourg, très concernés par le recours à la main-d'œuvre frontalière subissent les désagréments qui y sont liés : engorgements des principales voies d'accès et pollution notamment. Ajoutons aussi les impacts sur la pratique des langues, les risques potentiels pour la cohésion sociale, etc. 


\subsection{Le périmètre de la Grande Région SaarLorLux en question}

Dans un travail de zonage de l'espace régional lorrain, l'INSEE identifie cinq principaux pôles transfrontaliers qui s'étalent de part et d'autre des frontières nationales (cf. INSEE 2011):

- Longwy jusqu'à Aubange (Belgique) et Pétange, Esch-sur-Alzette et Luxembourg-ville (Luxembourg);

- Ottange jusque Kayl (Luxembourg) ;

- Saint-Avold jusque Lauterbach (Allemagne);

- Forbach jusque Sarrebrück (Allemagne);

- Sarreguemines jusque Habkirchen (Allemagne).

C'est au sein de ces territoires, compris dans une bande géographique de 20 à $30 \mathrm{~km}$ de part et d'autre des frontières, qu'a lieu la quasi-totalité des flux de travailleurs frontaliers résidant en Lorraine. Comme le fait observer l'INSEE, ces territoires «illustrent l'imbrication des agglomérations lorraines avec leurs voisines de la Grande Région et renvoient l'image d'un marché de travail transfrontalier devenu une réalité quotidienne pour 88000 Lorrains en 2008 (un peu plus de 100000 en 2011) ( (INSEE 2011: 2). Le phénomène de travail frontalier est relativement concentré géographiquement. Or la Grande Région SaarLorLux ne se résume pas à ces quelques territoires frontaliers. Elle comprend des espaces éloignés des frontières qui ne sont concernés que marginalement par les flux de travailleurs frontaliers. En lien avec cette question du périmètre de l'espace transfrontalier, les sentiments d'appartenance à ce grand espace sont très variables. Ils sont plus forts pour ceux qui habitent à proximité directe des frontières (cf. Soutif 1999), et naturellement pour ceux qui vivent au quotidien le travail frontalier, ces hybrides qui vivent entre deux pays, deux cultures comme les qualifiait Kessler (1991) ou Wille (2012). Quelle est alors la représentation de la Grande Région SaarLorLux pour un habitant d'Epinal, Charleroi ou encore Mayence?

\subsection{Des freins à la libre circulation des travailleurs}

Si nous ne pouvons pas parler de véritable processus d'intégration des territoires à la Grande Région SaarLorLux, c'est aussi parce que de nombreux obstacles à la mobilité des travailleurs persistent. Pourtant, les principes d'élimination des entraves à la libre circulation des travailleurs au sein de l'espace européen et l'interdiction de toute forme de discrimination entre travailleurs européens ont été affirmés avec force dès le Traité de Rome en 1957 et consacrés dans les règlements européens qui définissent la coordination des systèmes de sécurité 
sociale (cf. 1408/71; 883/2004). Mais l'affirmation de ces principes et les règlements européens qui en découlent ne règlent pas tous les problèmes liés à la mobilité des travailleurs. Réalisée dans le cadre d'un projet agréé par la Commission Européenne, une étude cerne les principaux freins (cf. CRD EURES Lorraine 2011). Sans les mentionner tous, nous en retiendrons quelques-uns.

L'absence de reconnaissance des diplômes, de concordance des qualifications, de référentiels sur les métiers peut par exemple constituer un sérieux frein à la mobilité transfrontalière. Seul un nombre restreint de métiers bénéficie aujourd'hui d'une reconnaissance des diplômes (cf. directive 2005/CE ; CRD EURES Lorraine 2011: 17). Par ailleurs, il est impossible pour un apprenti de travailler chez un employeur d'un autre pays, sauf si ce dernier accepte de payer la formation au centre du pays formateur. Précisons néanmoins sur ce sujet que le Luxembourg autorise depuis le règlement grand-ducal du 26 juillet 2010 l'apprentissage transfrontalier sur son territoire.

Ajoutons encore un frein lié à la détermination du lieu de résidence. Il n'est en effet pas possible d'être résident de plusieurs Etats-membres en même temps. Cette détermination est importante puisque les droits en matière de fiscalité en dépendent et, plus particulièrement, en Allemagne. Autre frein : la complexité du statut de travailleur intérimaire, lorsqu'il réalise des missions d'intérim de l'autre côté des frontières. En l'absence d'harmonisation des législations nationales de l'intérim, c'est une superposition de réglementations qui codifie ces relations de travail: celle du pays de localisation de l'entreprise de travail temporaire et celle du pays où se déroule la mission. Cette superposition est source de tensions qui fragilisent encore plus la situation de ces travailleurs déjà précaires (cf. Belkacem/Pigeron-Piroth 2014).

D'autres obstacles concernent également les conditions de déplacements des travailleurs frontaliers. Les embouteillages et les transports en commun surchargés sont le lot quotidien des travailleurs frontaliers. Pourtant de nombreux efforts ont été faits dans ce domaine comme la mise en place et le développement de lignes transfrontalières de bus, la réouverture de certaines haltes ferroviaires (p. ex. Hettange-Grande), l'augmentation de la capacité et du cadencement des trains qui assurent des liaisons frontalières. Mais cela ne semble pas encore suffire, surtout dans ce contexte de forte croissance du nombre de travailleurs frontaliers. Les autoroutes A31 et A30 (dans une moindre mesure) qui relient la Lorraine au Luxembourg et à la Belgique sont souvent surchargées. De plus l'A4 qui assure la liaison Metz/Sarrebruck est la seule autoroute payante de la région, ce qui accroît le coût de mobilité des travailleurs concernés. Les auteurs de l'étude CRD EURES Lorraine (2011) relèvent également des absences de liaisons directes par train entre Luxembourg-Ville et Sarrebruck, pourtant deux grandes métropoles de la Grande Région SaarLorLux. Il existe cependant une offre de bus (Express Sarrebruck) avec un système d'abonnement. 
Enfin, un dernier obstacle que l'on pourrait souligner ici concerne l'exercice de certains mandats, judiciaire ou politique. Il est par exemple impossible pour un travailleur frontalier d'exercer certains droits ou devoirs dans son pays de résidence comme juré en assise, conseiller communal ou encore de remplir ses obligations de réserviste de l'armée, car les facilités de temps ne leur sont pas accordées.

\subsection{Des points problématiques dans le statut du travailleur frontalier}

Nous pouvons également relever un certain nombre de points qui restent problématiques au sein-même du statut de travailleur frontalier : la reconnaissance des accidents du travail, la prise en compte de l'invalidité et les retraites.

La reconnaissance de l'accident du travail et de son degré de gravité fait l'objet de définitions différentes entre pays. Cette question est d'autant plus cruciale que certains travailleurs frontaliers, comme les intérimaires qui sont en mission de l'autre côté des frontières, connaissent des taux d'accident du travail comparativement plus élevés que les autres travailleurs frontaliers. Selon les statistiques officielles du Ministère du Travail français, de par la nature de leur activité professionnelle, les travailleurs intérimaires sont exposés à une fréquence d'accidents du travail deux fois plus forte que pour l'ensemble des salariés, parce qu'ils interviennent dans l'urgence le plus souvent et, dans des secteurs d'activité par nature très exposés comme celui de la construction ou de l'industrie (cf. Belkacem/Montcharmont 2012: 17). A ces questions qui touchent aux cadres institutionnels fort différents d'un pays à l'autre, s'ajoutent des questions plus pratiques, liées à la lourdeur administrative et aux temps d'attente très longs des inscriptions/désinscriptions aux divers organismes (sécurité sociale, chômage, etc.) auxquelles doivent se soumettre les travailleurs frontaliers qui disposent de contrats de travail successifs.

Un autre point épineux du statut des travailleurs frontaliers concerne la prise en compte de l'invalidité. Au sein de la Grande Région SaarLorLux, deux régimes d'invalidité sont d'usage. L'un prend en compte la durée d'affiliation (Luxembourg, Allemagne) et l'autre, la réalisation du risque (Belgique, France). Là encore, il n'existe pas de définition commune de l'invalidité et des taux d'invalidité. Si le critère retenu est celui de la durée d'affiliation, la pension d'invalidité est obtenue selon la même méthode que celle de la pension de vieillesse, c'està-dire selon la durée de cotisation. Dans la situation où la réalisation du risque est prise en compte, l'attribution de la pension d'invalidité est liée cette fois-ci au fait d'être assuré au moment où survient l'invalidité (cf. Borsenberger 2012 : 404). Les choses se compliquent lorsqu'une personne a été assurée successivement sous ces deux régimes. Ces différences de législations peuvent avoir des conséquences non seulement sur la reconnaissance de l’invalidité, mais égale- 
ment sur le montant du taux d'invalidité et par conséquent sur le montant de la pension elle-même.

A ces problèmes s'ajoute celui de l'âge légal de la retraite qui varie encore largement entre les pays. Pour les personnes ayant leur activité dans différents pays, comme c'est souvent le cas des intérimaires transfrontaliers, ceci est une importante source de difficultés au moment de la liquidation de la pension. Pour rappel, l'âge légal de départ à la retraite est fixé à 60 ans en France, (et à terme à $6_{2}$ ans). Il est de 65 ans en Allemagne, (et à terme $6_{7}$ ans), et de 65 ans au Luxembourg. Par ailleurs, concernant la portabilité des retraites complémentaires d'entreprise, il n'existe encore aujourd'hui aucun système de coordination.

De plus, l'imbrication des différents marchés se limite à l'emploi et n'inclut pas le chômage et la recherche d'emploi. Ainsi, la comptabilisation du chômage est nationale, car le frontalier qui perd son emploi, est pris en compte par l'administration de l'emploi de son pays de résidence et ce, même s'il recherche activement un emploi de l'autre côté des frontières. Les différentes administrations gérant des demandeurs d'emploi ne sont pas interconnectées et ne peuvent fournir des offres d'emploi que relatives à leur territoire d'action (cf. OREFQ 2011).

\subsection{Des avancées certaines}

Malgré ces obstacles et ces freins, qui limitent la libre circulation des travailleurs au sein de la Grande Région SaarLorLux, nous pouvons relever plusieurs initiatives qui témoignent d'une volonté de rapprochement et de collaboration de la part des territoires. En matière de formation professionnelle, un accord transfrontalier a été conclu et signé le 5 novembre 2014 : « [...] à travers le présent accord, les partenaires se fixent l'objectif de mieux se concerter sur la promotion de la formation professionnelle transfrontalière dans la Grande Région » (Accord-cadre relatif à la formation professionnelle transfrontalière dans la Grande Région). Cet accord vient renforcer l'accord transfrontalier pour la formation professionnelle signé entre la Sarre et la Lorraine en juin 2014 et qui concerne une quinzaine de formations professionnelles réalisables en alternance des deux côtés de la frontière depuis la rentrée de septembre 2014 .

Par ailleurs, en matière de placement des travailleurs, on peut également mentionner la création du service de placement transfrontalier de SarrebruckSarreguemines. L'objectif visé est d'améliorer la transparence sur le marché du travail, en rapprochant les besoins économiques sarrois des besoins d'emploi français. Une convention-cadre a été signée en février 2013 par les ministres français et allemand, puis une convention de partenariat entre Pôle Emploi et Bundesagentur für Arbeit en novembre 2013. Ce service, qui fonctionne depuis janvier 2014, rassemble huit conseillers bilingues de Pôle Emploi Sarregue- 
mines et Bundesagentur für Arbeit de Sarrebruck. Ils ont accès aux deux systèmes d'information et aux offres d'emploi des deux côtés de la frontière. En six mois, ce service de placement transfrontalier a dépassé ses objectifs annuels, d'accueil (300 personnes) et de placement (150 personnes).

\section{Conclusion}

Notre analyse s'est interrogée sur les fonctions du travail frontalier comme facteur d'intégration des différentes composantes régionales de la Grande Région SaarLorLux à un même espace transfrontalier. Elle délivre plusieurs enseignements qui permettent de proposer quelques éléments de réponse. Tout d'abord, la Grande Région SaarLorLux se vit et se pratique tous les jours par un grand nombre d'acteurs, à commencer par les travailleurs frontaliers. Ils constituent la preuve la plus visible de l'existence de cet espace transfrontalier et du lien qui existe entre les territoires qui le constituent. Phénomène ancien, conséquent et en forte croissance dans la Grande Région SaarLorLux, le travail frontalier n'a rien de conjoncturel. Il est au contraire durable et ancré dans les économies locales. Vecteur de rapprochement, il est aussi un révélateur des différences institutionnelles, économiques et politiques existant entre les territoires qui composent la Grande Région SaarLorLux. En permettant de résoudre un problème productif régional qui est, d'un côté des frontières, d'offrir un emploi aux personnes disponibles et, de l'autre côté des frontières, de trouver les qualifications, les compétences spécifiques nécessaires, il contribue au développement territorial des deux côtés des frontières. Il permet de rapprocher les différents territoires parce qu'il est aussi un construit social. Le travail frontalier est le résultat d'une codification par des règles juridiques nationales et supranationales, des conventions bilatérales entre pays et des accords, lui donnant une réalité et ayant favorisé son développement. Pour autant, la réalité d'un marché intégré reste encore limitée, par les freins à la libre circulation ainsi que les points problématiques en matière de statut (invalidité, retraite, etc.). Les derniers accords-cadres signés en matière de formation professionnelle et de placement transfrontalier ouvrent néanmoins des perspectives dans la construction d'un espace et d'un marché de l'emploi intégrés. Ils constituent en effet une avancée importante vers un rapprochement des systèmes de formation professionnelle et de placement de travailleurs. 


\section{Bibliographie}

Arrêté ministériel du 9 février 1921 établissant le régime de carte frontière et $\mathrm{du}$ visa collectif, Grand-Duché de Luxembourg.

Belkacem, Rachid/Montcharmont, Laurence (2012): Analyse des conditions de travail des travailleurs intérimaires: Comment expliquer la vulnérabilité des intérimaires aux accidents du travail ? Dans : PISTE - Perspectives interdisciplinaires sur le travail et la santé 14 (2), http://pistes.revues.org/2543 [consulté : 31-01-2015].

Belkacem, Rachid/Pigeron-Piroth, Isabelle (éd.; 2012a) : Le travail frontalier au sein de la Grande Région Saar-Lor-Lux. Pratiques, enjeux et perspectives. Nancy: PUN, Editions Universitaires de Lorraine.

Belkacem, Rachid/Pigeron-Piroth, Isabelle (2012b) : Les travailleurs frontaliers au sein de la Grande Région Saar-Lor-Lux. Dans : Idem (éd.) : Le travail frontalier au sein de la Grande Région Saar-Lor-Lux. Pratiques, enjeux et perspectives. Nancy: PUN, Editions Universitaires de Lorraine, p. 25-46.

Belkacem, Rachid/Pigeron-Piroth, Isabelle (2012c): Effet-frontière et développement transfrontalier. Dans: Idem (éd.) : Le travail frontalier au sein de la Grande Région Saar-Lor-Lux. Pratiques, enjeux et perspectives. Nancy: PUN, Editions Universitaires de Lorraine, p. 283-296.

Belkacem, Rachid/Pigeron-Piroth, Isabelle (2014) : Développement et diversification du travail frontalier: le cas des intérimaires transfrontaliers. $14^{\text {èmes }}$ Journées Internationales de Sociologie du Travail (17 au 19 juin 2014), Lille.

Borsenberger, Monique (2012) : Le statut juridique et conventionnel du travailleur frontalier. Dans: Rachid Belkacem/Isabelle Pigeron-Piroth (éd.): Le travail frontalier au sein de la Grande Région Saar-Lor-Lux. Pratiques, enjeux et perspectives. Nancy: PUN, Editions Universitaires de Lorraine, p. 397-409.

Convention entre la France et le Grand-Duché de Luxembourg, tendant à éviter les doubles impositions et à établir des règles d'assistance administrative réciproque en matière d'impôts sur le revenu et sur la fortune, version consolidée de la convention franco-luxembourgeoise du $1^{\mathrm{er}}$ avril 1958.

Convention entre le Luxembourg et la Belgique en vue d'éviter les doubles impositions et de régler certaines autres questions en matière d'impôts sur le revenu et la fortune du 17 septembre 1970 entre le Grand-Duché de Luxembourg et la République Fédérale d'Allemagne.

Convention entre le Luxembourg et la Belgique en vue d'éviter les doubles impositions et de régler certaines autres questions en matière d'impôts sur le revenu et la fortune du 23 août 1958 entre le Grand-Duché de Luxembourg et la République Fédérale d'Allemagne.

CRD EURES Lorraine (2011) : Freins à la mobilité des travailleurs frontaliers lorrains dans l'espace Lorraine/Luxembourg/Rhénanie-Palatinat/Sarre, Projet agréé par la Commission Européenne, Programme EURES-T SLLR 2010-2011. 
Davezies, Laurent/Talandier, Magali (2010): Les nouveaux enjeux du développement territorial: la structure économique des zones d'emploi françaises face au changement, Rapport à l'ADCF.

INSEE (2011): Le zonage en aires urbaines 2010: un éclairage sur notre nouvelle façon d'habiter et de vivre l'espace. Dans: Economie Lorraine 269, p. 1-7.

Kaufmann, Vincent (2008) : Les paradoxes de la mobilité, bouger, s'enraciner. Lausanne: Presses polytechniques et universitaires romandes.

Kessler, Simon (1991) : Frontaliers d'Europe. Rapport sur les migrations transfrontalières. Strasbourg: Ed'Image.

Löh, Christiane (2011) : Qui sont les travailleurs frontaliers de la Grande Région? Caractéristiques et déterminants de la mobilité professionnelle. Offices statistiques de la Grande Région.

Moreau, Gérard/Neiss, Martine (2007) : Le travail frontalier: l'âge de la maturité. Dans : Economie Lorraine 99, p. 1-10.

OIE (2012) : Situation du marché de l'emploi dans la Grande Région. Huitième rapport de l'Observatoire Interrégional du marché de l'Emploi pour le treizième Sommet des Exécutifs de la Grande Région. Sarrebruck.

OREFQ (2011): Defis pour le marché de l'emploi de la Grande Région. Etat des lieux et perspectives.

Pigeron-Piroth, Isabelle (2009) : Le secteur public. Dans : Economie et Statistiques 34, p. 1-34.

Pigeron-Piroth, Isabelle (2014): Multirésidences et travail frontalier au Luxembourg et dans la Grande Région Saar-Lor-Lux. Dans : Phillipe Hamman/Maurice Blanc/Cédric Duchêne-Lacroix/Tim Freytag/Catherine Kramer (éd.): Questionner les mobilités résidentielles à l'aune de la multilocalité. Strasbourg: Néothèque, p. 213-236.

Règlement grand-ducal du 26 juillet 2010 portant organisation de l'apprentissage transfrontalier.

Sauer, Arnaud/Scuto, Denis (2012): Le travail frontalier dans l'industrie lourde de l'entre-deux-guerres aux années 1970. Bilan historiographique, sources et pistes de recherche. Dans: Hémecht. Revue d'Histoire luxembourgeoise 64 (4), p. 75-98.

Schmitz, Frédéric/Drevon, Guillaume/Gerber, Phillipe (2012): La mobilité des frontaliers du Luxembourg. Dynamiques et perspectives. Dans: Les Cahiers du CEPS/Instead (numéro hors série).

Soutif, Véronique (1999) : L'intégration européenne et les travailleurs frontaliers de l'Europe Occidentale, Paris : L'Harmattan.

Thanry, Jean-Philippe (2012): Revenus des frontaliers lorrains du Luxembourg: des atouts tirés de la métropole luxembourgeoise. Dans: Economie Lorraine 280, p. 1-6.

Wille, Christian (2012) : Grenzgänger und Räume der Grenze. Raumkonstruktionen in der Großregion SaarLorLux, Francfort-sur-le-Main : Peter Lang.

Zanardelli, Mireille/Genevois, Anne-Sophie/Schuller, Guy (2012) : Les comportements de dépenses des salariés frontaliers sur le territoire luxembourgeois. 
Dans : Rachid Belkacem/Isabelle Pigeron-Piroth (éd.) : Le travail frontalier au sein de la Grande Région Saar-Lor-Lux. Pratiques, enjeux et perspectives. Nancy: PUN, Editions Universitaires de Lorraine, p. 47-70. 
